\title{
Trichome-Specific Expression of
}

\section{Amorpha-4,11-Diene Synthase, a Key Enzyme of Artemisinin Biosynthesis in Artemisia annua L., as Reported by a Promoter-GUS Fusion}

\author{
Hongzhen Wang, Linda Olofsson, Anneli Lundgren, Peter E. Brodelius \\ School of Natural Sciences, Linnaeus University, Kalmar, Sweden. \\ Email: peter.brodelius@lnu.se \\ Received August $16^{\text {th }}, 2011$; revised September $7^{\text {th }}, 2011$; accepted September $20^{\text {th }}, 2011$.
}

\begin{abstract}
Artemisia annua L. produces small amounts of the sesquiterpenoid artemisinin, which is used for treatment of malaria. A worldwide shortage of the drug has led to intense research to increase the yield of artemisinin in the plant. In order to study the regulation of expression of a key enzyme of artemisinin biosynthesis, the promoter region of the key enzyme amorpha-4,11-diene synthase (ADS) was cloned and fused with the $\beta$-glucuronidase (GUS) reporter gene. Transgenic plants of A. annua expressing this fusion were generated and studied. Transgenic plants expressing the GUS gene were used to establish the activity of the cloned promoter by a GUS activity staining procedure. GUS under the control of the $A D S$ promoter showed specific expression in glandular trichomes. The activity of the ADS promoter varies temporally and in old tissues essentially no GUS staining could be observed. The expression pattern of GUS and ADS in aerial parts of the transgenic plant was essentially the same indicating that the cis-elements controlling glandular trichome specific expression are included in the cloned promoter. However, some cis-element(s) that control expression in root and old leaf appears to be missing in the cloned promoter. Furthermore, qPCR was used to compare the activity of the wild-type ADS promoter with that of the cloned ADS promoter. The latter promoter showed a considerably lower activity than the wild-type promoter as judged from the levels of GUS and ADS transcripts, respectively, which may be due to the removal of an enhancing cis-element from the ADS promoter. The ADS gene is specifically expressed in stalk and secretory cells of glandular trichomes of A. annua.
\end{abstract}

Keywords: Agrobacterium Tumefaciens, Amorpha-4,11-Diene Synthase, Artemisia annua, Artemisinin Biosynthesis, $\beta$-Glucuronidase, Gene Regulation, Promoter Activity, Stable Transformation

\section{Introduction}

Artemisinin is an effective anti-malarial drug, which has become an important component of artemisinin-based combination therapies (ACTs) [1]. The content of artemisinin in Artemisia annua is, however, very low and ranges between $0.1 \%$ and $0.8 \%$ of dry weight [2]. Selection of high-producing varieties of $A$. annua has met limited success but hybrids producing increased amounts of artemisinin have been produced [3]. However, there is still a shortage in the supply of artemisinin [4]. Different methods have been tried to improve the artemisinin production such as treatment with abscisic acid [5], gibberelic acid [6-8] and elicitors [9-11]. Various transgenic A. annua plants have been produced to increase the yield of artemisinin. These attempts include down-regulation of squalene synthase [12,13] or up-regulation of farnesyl diphosphate synthase (FDS) [14].

Cyclization of farnesyl diphosphate (FDP) to amorpha-4,11-diene by amorpha-4,11-diene synthase (ADS) is the initial step of the artemisinin biosynthetic pathway [15] and amorpha-4,11-diene is the committed precursor [16]. Tissue specificity of ADS expression has been shown by GUS expression in Arabidopsis thaliana using a fusion of the ADS promoter and the reporter gene [17]. In the following step of the artemisinin biosynthesis, amorpha-4,11-diene is hydroxylated to yield artemisinic alcohol. This reaction is catalyzed by a cytochrome P450 dependent amorpha-4,11-diene 12-hydroxylase (CYP71AV1) 
[18]. This enzyme is multifunctional and can also oxidize the alcohol to artemisinic aldehyde and then further on to artemisinic acid [19]. However, artemisinic acid is a direct precursor of arteannuin B biosynthesis. For artemisinin biosynthesis the artemisinic aldehyde must be reduced to dihydroartemisinic aldehyde, which subsequently is oxidized to dihydroartemisinic acid and then converted to artemisinin [20]. These two steps are catalyzed by artemisinic aldehyde $\Delta 11$ (13) reductase [21] and aldehyde dehydrogenase 1 [22], respectively. Glandular trichome specific expression of ADS and CYP71AV1 $[17,18]$ strongly supported the notion that artemisinin is sequestered and localized to glandular trichomes of A. апnиa $[23,24]$.

In order to understand the mechanisms behind the increase in artemisinin biosynthesis after treatment with hormones [5-8] or biotic elicitors [9-11], a detailed knowledge about the regulation of expression of different enzymes involved in artemisinin biosynthesis will be essential. To our knowledge, there is no data available on the regulation of expression of biosynthetic genes in $A$. annua using promoter-reporter gene fusions in transgenic plants of A. annua. We have initiated studies on the regulation of terpene metabolism in different tissues of $A$. annua [25] and here we report on results from transgenic A. annua expressing a fusion of the reporter gene (GUS) and the promoter of the key enzyme ADS.

\section{Materials and Methods}

\subsection{Plant Materials}

Plants of A. annua were grown under $16 \mathrm{~h}$ days and $8 \mathrm{~h}$ nights at $22^{\circ} \mathrm{C}$ to a height of approximately $1 \mathrm{~m}$ followed by induction of flowering at $8 \mathrm{~h}$ days and $16 \mathrm{~h}$ nights at $22^{\circ} \mathrm{C}$. Flower buds, young leaves, old leaves, stems and roots were collected for GUS staining. Samples of these tissues were also frozen in liquid nitrogen, ground and used for RNA extraction.

\subsection{Promoter Cloning}

Genomic DNA was extracted from fresh young leaves of A. annua using the CTAB method [26]. The promoter sequence of ADS was available in the GenBank (accession number AY528931). The sequence was used to design primers for PCR amplification (primers 1 and 2 in Table 1) of a 1929 bp fragment of the ADS promoter using Pfu polymerase (Fermentas). Primers 1 and 2 carried $\mathrm{XmaI}$ and $\mathrm{NcoI}$ restriction site, respectively, used for cloning the fragment into the plant transformation vector.

\subsection{Construction of Transformation Vector}

The pCAMBIA $1381 \mathrm{Z}$ vector (CambiaLabs, Brisbane,
Table 1. Nucleotide sequence of primers used. Restriction sites are underlined; $\mathbf{f}=$ forward; $r$ = reverse.

\begin{tabular}{ccc}
\hline Primer & Name & Primer Sequence \\
\hline 1 & $p A D S_{\mathrm{f}}$ & TCCCCCGGGAAAGTTAATTGCACATAT \\
2 & $p A D S_{\mathrm{r}}$ & CATGCCATGGATTTTCAAAACTTTGAATAT \\
3 & GUS $_{\mathrm{f}}$ & AACCGTTCTACTTTACTGGCTTTGG \\
4 & GUS $_{\mathrm{r}}$ & GCATCTCTTCAGCGTAAGGGTAAT \\
5 & $A D S_{\mathrm{f}}$ & GGGAGATCAGTTTCTCATCTATGAA \\
6 & $A D S_{\mathrm{r}}$ & CTTTTAGTAGTTGCCGCACTTCTT \\
7 & $\beta-$ actin $_{\mathrm{f}}$ & CCAGGCTGTTCAGTCTCTGTAT \\
8 & $\beta-$ actin $_{\mathrm{r}}$ & CGCTCGGTAAGGATCTTCATCA \\
\hline
\end{tabular}

Australia) carrying the GUS-gene was used for the transformation of A. annua. However, the vector was modified in that the plant resistance gene was changed from hygromycin to kanamycin. An XhoI fragment $(0.88 \mathrm{~kb})$ carrying the NPTII gene was cut out from pCAMBIA 2301 and ligated into pCAMBIA 1381Z digested with XhoI. Restriction analysis was used to determine that the XhoI fragment had been ligated in the right direction.

The ADS promoter was double-digested with NcoI/ XmaI and inserted into the modified pCAMBIA $1381 \mathrm{Z}$ vector digested with the same restriction enzymes. The plant transformation vector obtained (pCAMBIA1381ZpADS-GUS) was introduced into E. coli BL21, which was grown on LB medium containing kanamycin (50 $\mathrm{mg} / \mathrm{L}$ ). The vector was purified using the GeneJet Plasmid Miniprep Kit (Fermentas) and used for transformation of Agrobacetrium tumefaciens EHA105.

The plant transformation vector was introduced into $A$. tumefaciens EHA105 by the freeze and thaw method. A. tumefaciens EHA105 carrying the plant transformation vector was grown on YEP medium containing kanamycin $(100 \mathrm{mg} / \mathrm{L})$, rifampicin $(40 \mathrm{mg} / \mathrm{L})$ and streptomycin $(25 \mathrm{mg} / \mathrm{L})$ at $28^{\circ} \mathrm{C}$ to an $\mathrm{OD}_{600}=0.8-1$. The cells were collected by centrifugation and resuspended in MSMO liquid medium to an $\mathrm{OD}_{600}=0.3-0.5$.

\subsection{Plant Transformation}

A. annua seeds (variety Chongqin) were immersed in $75 \%$ ethanol for 1 min, surfaced sterilized with $5 \%$ sodium hypochlorite $(\mathrm{NaOCl})$ for 20 min, washed $3-4$ times with sterile distilled water. The sterilized seeds were germinated on solid MSMO medium (Sigma) (MSMO powder $4.4 \mathrm{~g} / \mathrm{L}$, sucrose $30 \mathrm{~g} / \mathrm{L}$, agar $8-9 \mathrm{~g} / \mathrm{L}$, $\mathrm{pH}=5.8$ ) at $28^{\circ} \mathrm{C}$ for $20-25$ days. The leaflets of $20-25$ days old seedlings were used as explants for transformation. The explants were immersed into a suspension of $A$. tumefaciens EHA105 carrying the pCAMBIA1381ZpADS-GUS transformation vector. After $30 \mathrm{~min}$ the explants were transferred to co-cultivation medium (MSMO 
medium containing 6-BA $(0.5 \mathrm{mg} / \mathrm{L})$, NAA $(0.05 \mathrm{mg} / \mathrm{L}))$ and the A. tumefaciens EHA105. Co-cultivation was carried out in darkness at $28^{\circ} \mathrm{C}$ for $48 \mathrm{~h}$. Subsequently, the explants were transferred to selection and germination medium (MSMO medium containing 6-BA $(0.5 \mathrm{mg} / \mathrm{L})$, NAA $(0.05 \mathrm{mg} / \mathrm{L})$, kanamycin $(100 \mathrm{mg} / \mathrm{L})$ and carbenicillin $(250 \mathrm{mg} / \mathrm{L}))$. The explants were transferred to new medium every second week. Shoots started to grow out after around two weeks. The shoots were transferred to root inducing medium (half strength MSMO medium containing kanamycin $(100 \mathrm{mg} / \mathrm{L})$ and carbenicillin $(100$ $\mathrm{mg} / \mathrm{L})$ ). After rooting the plantlets were left 4 weeks before they were transferred to soil.

\subsection{Scanning Electron Microscopy}

Scanning electron microscopy (SEM) was carried out on unfixed tissues from wild-type and transgenic $A$. annua plants using a relatively low energy beam $(5 \mathrm{kV})$ on a LEO 435VP scanning electron microscope.

\subsection{GUS Assay}

Leaf primordia at the apex and expanded leaves at different nodes, stems, roots from shoots and flowering plants, and flower buds were sampled from transgenic $A$. annua plants to perform GUS analysis. GUS histochemical staining of the various plant tissues was carried out as previously described [27]. GUS stained tissues were studied under a microscope (Nikon ECLIPSE E400) and photographs were taken using a digital camera (Nikon DP11).

\section{7. qPCR}

Different tissues of transgenic A. annua plants were sampled to analyze the relative expression pattern of $p A D S$-GUS and $p A D S$ - $A D S$ using $\beta$-actin as the reference gene. RNA was extracted using Purelink ${ }^{\mathrm{TM}}$ Plant RNA Reagent (Invitrogen, Carlsbad, CA, USA) according to the manufacturer's instruction. Genomic DNA was removed by treatment with DNase I (Fermentas, St LeoRoth, Germany). RNA (1 $\mu \mathrm{g})$ was reverse transcribed using RevertAid ${ }^{\mathrm{TM}} \mathrm{H}$ Minus-MuLV reverse transcriptase (Fermentas) primed with $0.5 \mu \mathrm{g}$ oligo $(\mathrm{dT})_{18}$ primer. RNA was removed from the cDNA obtained by treatment with RNase $\mathrm{H}$ (Fermentas).

The qPCR was performed on a 7500 qPCR thermocycler (Applied Biosystems, Foster City, USA) using primers listed in Table 1 for GUS (primers 3 and 4), ADS (primers 5 and 6 ) or actin (primers 7 and 8). First singlestranded cDNA was used as template in a $20 \mu \mathrm{l}$ reaction mixture containing $10 \mu \mathrm{l}$ Power SYBR $^{\circledR}$ Green PCR Master Mix (Applied Biosystems) and 2 pmol of each primer. The qPCR thermal cycling was performed at $50^{\circ} \mathrm{C}(2 \mathrm{~min}), 95^{\circ} \mathrm{C}(10 \mathrm{~min}), 40$ cycles at $95^{\circ} \mathrm{C}(15 \mathrm{sec})$, $60^{\circ} \mathrm{C}(1 \mathrm{~min})$ and finally a dissociation state at $95^{\circ} \mathrm{C}(15$ $\mathrm{sec}), 60^{\circ} \mathrm{C}(1 \mathrm{~min})$ and $95^{\circ} \mathrm{C}(15 \mathrm{sec})$. Triplet samples were run for each cDNA sample.

\subsection{Statistical Methods}

Grubbs test ( $\mathrm{G}=\mid$ Suspect value- $\left.\mathrm{x}_{\text {mean }} \mid / \mathrm{s}\right)$ was used to test for outliers. The outliers were rejected if $G_{\text {calculated }}>$ $\mathrm{G}_{\text {critical }}$ at $\mathrm{P}=0.05$. The critical value of $\mathrm{G}$ is 1.155 when the sample size is three [19].

\section{Results and Discussion}

\subsection{Cloning of Promoter of ADS}

Based on the sequence of the promoter region of ADS (GenBank accession number AY528931), a promoter region of 1929 bp upstream of the translation initial codon (ATG) as shown in Figure 1 was isolated from A. annua genomic DNA by PCR using high fidelity polymerase and primers 1 and 2 as listed in Table $\mathbf{1 .}$

\subsection{Prediction of Transcription Start Site and Core Promoter Elements}

The transcription start site (TSS) of the cloned promoter was predicted using the TSSP software (http://linux1. softberry.com/berry.phtml). A putative TSS of ADS (labeled +1 in Figure 1) was predicted 51 bp upstream of the translation initiation ATG-codon.

The TATA-box is a cis-regulatory element found in the promoter region of many genes in eukaryotes with the core sequence or a variant, which is usually followed by three or more adenine bases. It is considered to be the core promoter sequence and the binding site of either general transcription factors or histones (the binding of a transcription factor blocks the binding of a histone and vice versa). It is involved in the process of transcription by RNA polymerase and is usually located around $25 \mathrm{bp}$ upstream of the TSS. A putative TATA-box was found at positions -29 to -24 (TATAAA) in the ADS promoter (Figure 1) upstream of the putative TSS.

The CAAT-box signal is the binding site for the RNA transcription factor, and is typically accompanied by a conserved consensus sequence (GGCCAATCT). Genes that have this element seem to require it for the gene to be transcribed in sufficient quantities. This box along with the GC-box is known for binding general transcription factors. CAAT- and GC-boxes are primarily located in the region from 100 - 150 bp upstream from the TATA-box. Binding of specific protein is required for the CAAT-box activation. These proteins are known as CAAT-box binding proteins/CAAT-box binding factors. We could not find any CAAT-box with the consensus 
-1878 AGTTAATTGCACATATATGCTGAAAGTTGGCGTTGGTTTAACCCATTTAAACACTTCAGAAAAATCCACAAAAATTCCAGGAAGTCACAAATAACCCAAA -1779 TCAATTAACGTGTATATACGACTTTCAACCGCAACCAAATTGGGTAAATTTGTGAAGTCTTTTTAGGTGTTTTTAAGGTCCTTCAGTGTTTATTGGGTTT

-1778 TATTACTCCTGAAAAAGGAGAGCTCCTAAACCCCTGTGGTTTGAAAATGAAAAATCAAAAACACCATCTGAATTTTCAGACTCATAAACTCAGGATTGAT -1679 ATAATGAGGACTTTTTCCTCTCGAGGATTTGGGGACACAAACTTTTACTTTTTAGTTTTTGTGGTAGACTTAAAAGTCTGAGTATTTGAGTCCTAACTA

-1678 AACCAATGTTCTAAAAATCAGAAAGCTTACCTCCCGACCTCTCATGACCATGTAACTTACATATTCAGAAATTATACAGAAAGTACTCATGTTTAGACTC -1579

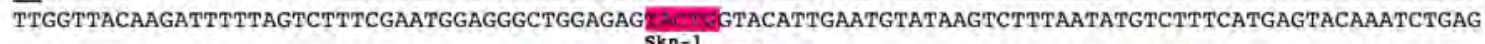

-1578 AAGAATCGCCCAAAAACACATTTCTATCATTAACTTATGACCCTTGCAAGACGACCCATCTCCTGGACAGCATGCAGTTGAGACAGAAACAAACCCTAAC -1479 TTCTTAGCGGGTTTTTGTGTAAAGATAGTAATTGAA GGACGTTGTGCTGGGTAGAGGACCTGTCGTACGTCAACTCTGTCTTTGTTTGGGATT

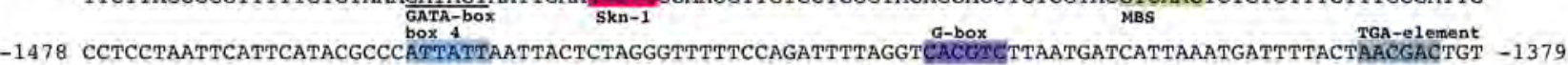
GGAGGATTAAGTAAGTATGCGGGTAATAATTAATGAGATCCCAAAAAGGTCTAAAATCCAGTGCAGAATTACTAGTAATTTACTAAAATGATTGCTGACA

GARE-motif AE-box CBPHV
-1378 ATTCCCCATTGATTCAAATGACAARAACAGAAACAAGGTCGACCCATAGGATTATGGTTTCATGATTTTAGGTCTTGTGTTTCAATTCTTATGACTTGGT -1279 TAAGGGTAACTAAGTT Skn-1 INTTTGTCTTTGTTCCAGCTGGGTATCCTAATACCAAAGTACTAAAATCCAGAACACAAAGTTAAGAA
GT1-mot if

-1278 ACATGTTATAACAATCCACAAATGTGCTTAAACAACCATTATAGCGTGACATGAATTAAAGGAAAT GAGAAGAAAAAAAATAGAATCGTTACCCCTTTAT -1179 TGTACAATATTGTTAGGTGTTTACACGAATTTGTTGGTAATATCGCACTGTACTTAATTTCCTTTACTCTICTTTTTTTTATCTTAGCAATGGGGAAATA py-rich region

-1178 TTTTGTGTCGAATGAAAATGAAATCGTGAGCTCTATAGGAATTCGCACTACACACATGGACCGAATCAAAATGCATCCTAGCCTTCTTGATTGATCAC - 1079 AAAACACAGCTTACTTTTACTTTAGCACTCGAGATATCCTTAAGGCGTGATGTGTGTACCTGGCTTAAGTTTTACGTAGGATCGGAAGAACTAACTAGTG GATA-boX AU-RR core

-1078 CACATCGAACGGGAGGAAGAAAAGCAGCTTGGCCTCATTTTGGTGGAGATAAAAGTTCATACGCATTTTTATTTTGATGTTAGGGTTTTGAGAACA ACE GTGTAGCTTGCCETCCTICTTTTCGTCGAACCGGAGTAAAACCACCTCTATTTTCAAGTATGCGTAAAAATAAAACTACAATCCCAAAACTCTTGTTTTG $\mathrm{Sp} 1$

-978 CGGTTATGTATTATTTTGATGGACTTGGGCTTGGGAAAAATTTAATGGGTCAATTGGGGAGTGTTTAGAAAGGCCCAATGGATTTTAGTTGGGGTTAGTT GCCAATACATAATAAAACTACCTGAACCCGAACCCTTTTTAAATTACCCAGTTAACCCCTCACAAATCTTTCCGGGTTACCTAAAATCAACCCCAATCAA w1-box

-878 TAGTCCGTGTAGCTTGTGTCGAATAATCGTGGCCCAAAAACAATTATCGGGTTTCGTTTAAATAATTATCGTAGCCCAAATTTAATTTACAAAAATTAAT ATCAGGCACATCGAACACAGCTTATTAGCACCGGTTTTTGTTAATAGCCCAAAGCAAATTTATTAATAGCATCGGGTTAAATTAAATGTTTTTAATTA GATA-boN GATA-bOX

-778 TACGGCTTATAATGACATGTTTGGTTCAAAATTTTAATTATATCACATATTATACATATTACGGGTCGTAGTGAAATTGTTTAACGGTTTACGAAATTCG ATGCCGAATA GL GTACAACCAAGTTTAAAATTAAATAGTGTATAATATGTATAATGCCCAGCATCACTTTAACAAATTGCCAAATGCTTTAAGC

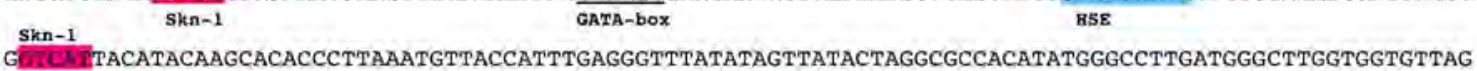
CCAGTAATGTATGTTCGTGTGGGAATTTACAATGGTAAACTCCCAAATATATCAATATGATCCGCGGTGTATACCCGGAACTACCCGAACCACCACAATC

-578 ACTTGCATTGTTTACACTTTTATTACAAGCTTACACTATCCTAACACATTATCTATCATCATACAATGTCCATAGCCCGATACTACTTTAATACAATTA TGAACGTAACAAAATGTGAAATAATGTTCGATGTGATAGGATTGTGTAATAGATAGTAGTATGTTACAGGTATCGGGCTATGATGAAATTATGTTAAT

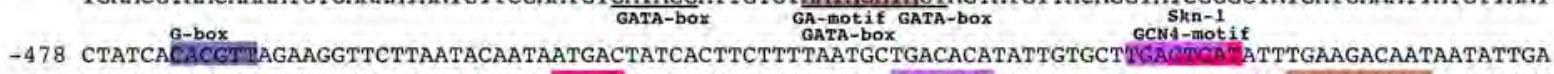

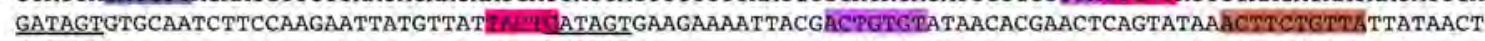
GATA-box Skn-1 GATA-box GARE GCN-motif GATA-box TC-rich repeats

-378 TTACATTTGATCTTAAATTGTAGTTAGAAATARE AAGAAAACATGGATCGGTTGTTTTATAGGATATAGGACAACATGTAGATTTAATAAATATTGTGAG AATGTAAACTAGAATTTAACATCAATCTTTATTGTTCTTTTGTACCTAGCCAACAAAATATCCTATATCCTGTTGTACATCTAAATTATTTATAACACTC

$$
\begin{aligned}
& \text { W1-box CGCG-box } \\
& \text { GTTGACCCATTCATCACGCGGGCC }
\end{aligned}
$$

-278 TAAGACTAATATGTTTTTGAAATGTTGACCCATTCATCACGCGGGCCGTGTTGGCTAGTGTTAAAGTAATACATTGTTTAATAAAATTCAATTGTTTACC ATTCTGATTATACAAAAACTTTACAACTGGGTAAGTAGTGCGCCCGGCACAACCGATCACAATTTCATTATGTAACAAATTATTTTAAGTTAACAAATGG TCT-motif box 1 ERE

CAAT-box
-178 GGTTGTATGAAAATATTTTACCCCGTAAAAAAAAATCAATTGTTMGGTTTAGGTCTTAGTGAGTGTGCTACTGTCAAAATATTTTACCCCATAAAAAA CCAACATACTTTTATAAAATGGGGCATTTTTTTTTAGTTAACAAATCCTAAATCCAGAATCACTCACACGATGACAGTTTTATAAAATGGGTATTTTTT CAAT-box

TATA-bo
-78 AATTCAT-box
\$1 TTAAGTTAACAAATCCTAAATTCAGAATAACTCACACGATGACAGAAAGATATTTTAGATCATTGAGAGGTAGTTCGATTCTCGTTGAGATCATTTAATT

23 ACCACATATATTCAAAGTTTTGAAAATCOATG TGGTGTATATAAGTTTCAAAACTTTTAGGTAC

Figure 1. Nucleotide sequence of the cloned ADS promoter with putative cis-elements shown.

sequence GGCCAATCT. However, CAAT-box sequences may differ considerably from the consensus sequence as exemplified by the promoters of the EAS3 and EAS4 genes encoding the sesquiterpene synthase epiaristolochene synthase in Nicotiana tabacum [28], which were determined to be GATCAATTA for both promoters.
Two putative CAAT- boxes were identified within 150 bp upstream of the TATA-box of the ADS promoter as shown in Figure 1 (e.g. AATCAATTG orATTCAATTG at positions -145 to -137 and -77 to -69 upstream of the TSS, respectively). It may be pointed out that by inspecting the promoter sequences for TATA- and CAAT- 
boxes a number of alternative putative TSSs may be found.

\subsection{Prediction of Cis-Elements of Promoter Region of ADS}

Putative cis-elements of the promoter were predicted by using the PLANTCARE software (http://bioinformatics. psb.ugent.be/webtools/plantcare/html/).

The py-rich sequence TTTCTTCTC is a cis-acting element conferring high transcription levels. Such an element was found at position -1204 to -1212 as shown in Figure 1.

Yang and Poovaiah (2000) reported that the tobacco ethylene-responsive gene NtER1 encodes a calmodulinbinding protein [29] and demonstrated later that there is one NtER1 homolog as well as five related genes in Arabidopsis [30]. These six Arabidopsis genes are rapidly and differentially induced by environmental signals such as extreme temperatures, UV-B, salt, wounding, hormones such as ethylene and abscisic acid (ABA), and signal molecules such as methyl jasmonate (MeJA), $\mathrm{H}_{2} \mathrm{O}_{2}$, and salicylic acid (SA). Hence, they were designnated as AtSR1 to 6 (Arabidopsis thaliana signal-responsive genes). AtSR1 targets the nucleus and specifically recognizes a 6-bp CGCG-box (A/C/G)CGCG(G/T/ C). Multiple CGCG cis-elements are found in promoters of genes involved in ethylene, ABA and SA signaling, and light signal perception. A putative CGCG-box at positions -240 to -235 was found in the ADS promoter (Figure 1).

The TCA-element with the sequence GAGAAGAATA is involved in responsiveness of genes to SA [31]. One such element with the sequence GAGAAGAAAA $(-1212$ to -1203) was found in the ADS promoter (Figure 1). A putative ethylene responsiveness ERE-element (ATTTCAAA) was localized to -256 to -268 . Furthermore, a putative gibberellin (GA)-responsive element (the GARE-motif AAACAGA) is present in the ADS promoter at position -1354 to -1348 and a modified GAREmotif (TAACAAG) at position -348 to -342 . A TC-rich repeat, which is involved in defense and stress response, was localized to position -387 to -396 (ATTGTCTTCA). Finally, an AU-RR core sequence (GGTCCAT), which is involved in auxin responsiveness, was found at position -1116 to -1122 .

The elements described above may be involved in the response of A. annua to plant hormones (ABA, SA, ethylene, MeJA and GA). In fact, treatment of $A$. annua plants with ABA $(10 \mu \mathrm{M})$ resulted in the induction of a number of genes encoding enzymes involved in artemisinin biosynthesis [5]. The expression levels of 3-hydroxy-3-methylglutaryl coenzyme A reductase (HMGR), farnesyl diphosphate synthase (FDS), CYP71AV1 and cytochrome P450 reductase (CPR) were significantly induced while ADS only showed a slight increase. The treatment with ABA resulted in a $65 \%$ increase in artemisinin content in A. annua plants [5].

Treatment of $A$. annua plants with $1 \mathrm{mM}$ SA resulted in a gradual increase in the expression of the HMGR gene and a temporary peak in the expression of the ADS gene [32]. The expression of the FDS and CYP71AV1 genes showed little change. At $96 \mathrm{~h}$ after SA treatment, the concentration of artemisinin, artemisinic acid and dihydroartemisinic acid were $54 \%, 127 \%$ and $72 \%$ higher than that of the control, respectively [32].

In a study on the production of artemisinin in cell suspension cultures of $A$. annua, acetyl-SA ( $20 \mathrm{mg} / \mathrm{L}$ ), JA (5 $\mathrm{mg} / \mathrm{L})$ or $\mathrm{GA}(10 \mathrm{mg} / \mathrm{L})$ were efficient elicitors and gave around 2-, 4- and 2.5-fold increased yield of artemisinin, respectively [10], indicating an increased expression of the key regulatory enzyme ADS. The beneficial effect of $\mathrm{GA}_{3}$ on artemisinin accumulation is also supported by the findings that treatment of $A$. annua plants with halfstrength Hoagland's solution containing $14 \mathrm{mM} \mathrm{GA}_{3}$ increased artemisinin content from $0.14 \%$ to $0.64 \%$ (w/w) when applied to 74-day-old plants [8]. No studies on the effects of GA on the expression level of enzymes involved in artemisinin biosynthesis have been reported.

Two W1-boxes ((C/T)TGAC(C/T)), defined as elicittor-responsive elements, have been identified at positions -255 to -250 and -926 to -931 (Figure 1). The transcription factor WRKY binds to these elements triggering the transcription of the gene. Transient expression of the A. annua transcription factor AaWRKY in agroinfiltrated leaves of $A$. annua resulted in increased levels of HMGR, ADS, CYP71AV1 and DBR2 indicating that several of the genes encoding enzymes involved in artemisinin biosynthesis are induced by binding of AaWRKY to the W-boxes [33]. In cotton, the GaWRKY1 is regulating the activity of cadinene synthase 1 (CAD1) involved in the biosynthesis of sesquiterpene phytoalexins [34]. The CAD1 promoter carries two W-boxes to which the GaWRKY1 binds. A recent study showed that foliar application of an elicitor (chitosan) resulted in increase of dihydroartemisinic acid and artemisinin by $72 \%$ and 53\%, respectively, in A. annua [11]. Furthermore, semi-quantitative RT-PCR showed an increased level of ADS transcripts $2 \mathrm{~h}$ after application of chitosan.

GATA-factors, involved in light-mediated regulation, are a class of transcriptional regulators present in plants that normally recognize the consensus sequence (T/A) GATA(G/A) [35]. Twelve putative GATA-boxes were found in the promoter region of ADS as shown in Figure 1. Other elements putatively involved in light-mediated 
regulation are the ACE-motif (AAACCGGTTA; position -982 to -973), the AE-box (AGAAACAA; position -1350 to -1343 ), the Box 1 (TTTCAAA; position -257 to -263 and +47 to +41 ), the Box 4 (ATTAAT; positions -784 to -779 and -1455 to -1450 ), the G-box (CACGT (T/C); position -1529 to $-1524,-1418$ to -1413 and -472 to -467 ), the GT1-motif (AATCCACA; position -1266 to -1259$)$, the Sp1-motif (CC(G/A)CCC; position -1063 to -1068 ) and the TCT-motif (TCTTAC; position -274 to -279$)$.

Two putative MYB binding sites (MBS) (TAACNG) at positions -697 to -692 and -1499 to -1504 were found in the ADS promoter. In Arabidopsis, they are the binding site for the myb proteins ATMYB1 and ATMYB2 involved in regulation of genes that are responsive to water stress [36] and in Petunia hybrida for MYB.Ph3 involved in regulation of flavonoid biosynthesis [37]. Twenty-eight MYB transcription factors have been identified in A. annua according to the plant transcription factor database (http://planttfdb.cbi.pku.edu.cn/ index.php? sp=Aan). To our knowledge, no study on the effects of MYB transcription factors on metabolism in A. annua has been reported.

A number of amorphane sesquiterpenes have been isolated from seeds of $A$. annua including artemisinic acid, dihydroartemisinic acid and arteannuin B indicating that enzymes of artemisinin biosynthesis are expressed in seeds [38]. These results have been questioned since there are no trichomes on seeds [39]. However, the ADS promoter contains a number of putative cis-elements involved in endosperm expression. These include eight Skn-1 (GTCAT) and two GCN4-motifs (TG(T/A)GTCA). Consequently, ADS may be expressed and amorphane sesquiterpenes produced in seeds of $A$. апnиa even though no trichomes are present. Finally, two HSE elements (AAAAAATTTC), which are involved in response to heat, were localized to positions -698 to -706 and -83 to -74 and one CBFHV element (GTCGAC), involved in salt and dehydration stress, at position -1341 to -1336 .

\subsection{Activity of the ADS Promoter}

In order to study the temporal and spatial expression of $A D S$, the cloned $A D S$ promoter was inserted into the modified (kanamycin) pCAMBIA $1381 \mathrm{Z}$ plant transformation vector carrying the GUS reporter gene. Transgenic $A$. annua plants carrying the fusion of the $A D S$ promoter and the GUS gene were produced using Agrobacterium tumefaciens. Twenty-seven positive $\mathrm{T}_{0}$ transgenic lines were selected by PCR. Fifteen of these $\mathrm{T}_{0}$ transgenic lines showed similar GUS staining pattern while the other twelve lines did not show any obvious GUS staining, which may be due to silencing of the introduced GUS gene.

There are two kinds of trichomes on aerial organs of $A$. annua, i.e. glandular secretory trichomes (GSTs) and non-glandular T-shaped trichomes (TST) (Figure 2). GUS staining was observed in GSTs of leaves (Figure 3(a)), stems of one-week old plantlets after root initiation (Figure 3(b)), leaf primordia (Figure 3(d)) and flower buds (Figure 3(e) and (f)). No GUS staining was observed in roots of plantlets (Figure 3(c)) but GUS staining was observed in roots at the reproductive stage. Furthermore, no GUS staining was observed in TSTs on leaves (Figure 3(g)). Based on data from the promoter/reporter fusion studies, it appears that ADS, the key enzyme of artemisinin biosynthesis, is almost exclusively expressed in GSTs and consequently the biosynthesis of artemisinin precursors is localized to this type of trichomes. We have previously reported that ADS only is expressed in apical cells of glandular trichomes [40]. However, the results obtained here clearly shows that the ADS promoter also is active in sub-apical cells (Figure 3(h)). This difference may be due to fixation of the tissue with formaldehyde before isolation of the apical cells by laser microdissection [40]. In a recent study, we have shown that isolation of RNA from formaldehyde-fixed cells is problematic and difficult to do in a reproducible way [L. Olofsson et al., unpublished]. However, isolation of RNA from unfixed cells with subsequent amplification is a good

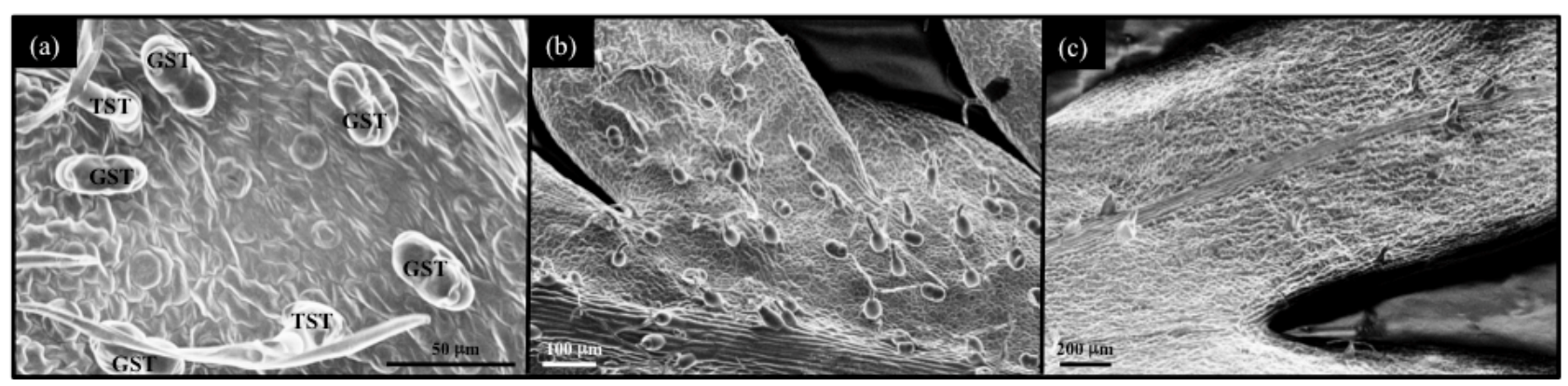

Figure 2. Scanning electron microscopy of A. annua. (a) close-up of young leaf; (b) young leaf; (c) old leaf (leaf before senescence). GST: glandular secretory trichome; TST: T-shaped trichome. 


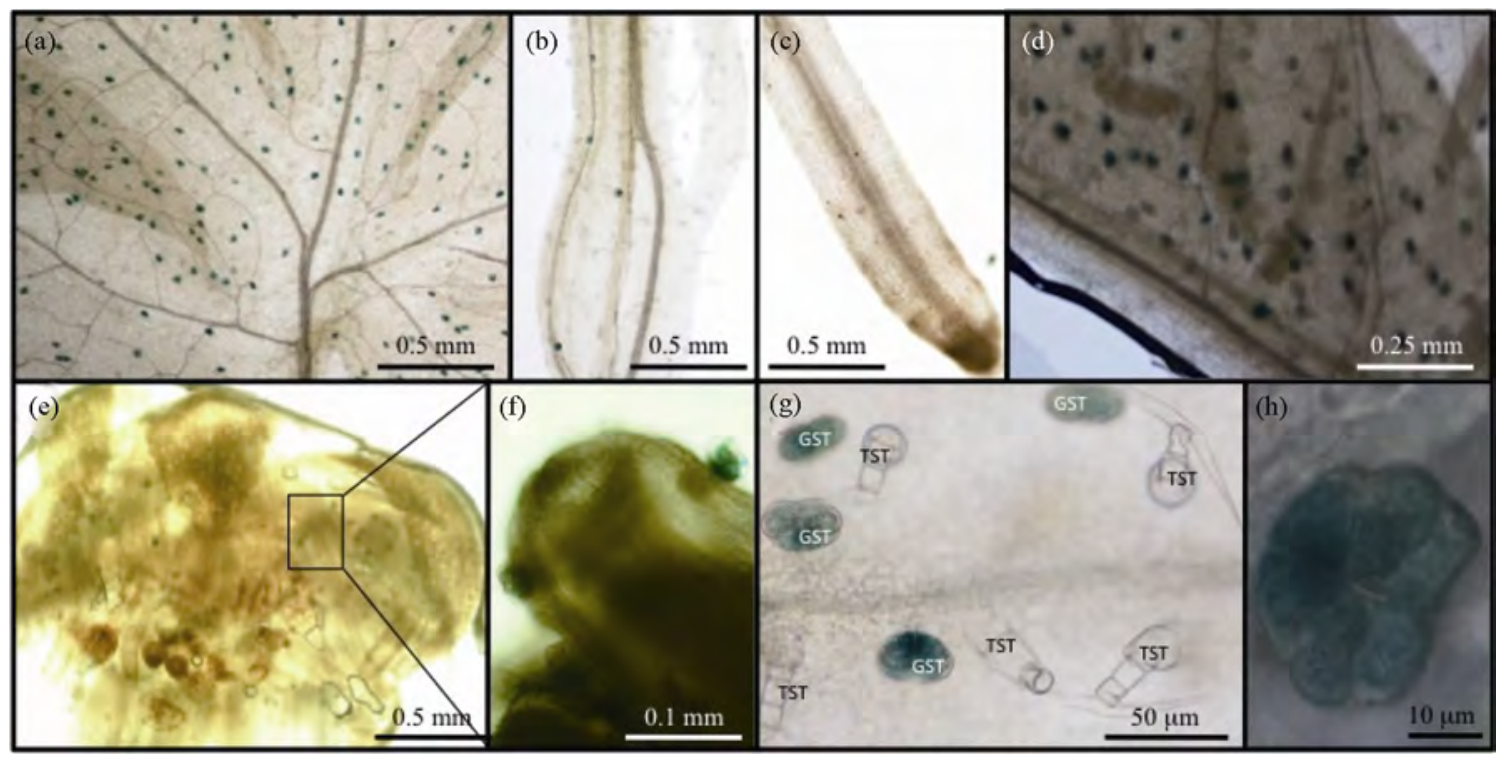

Figure 3. Gus-staining of transgenic A. annua transformed using the pADS-GUS plasmid. (a) leaf; (b) stem; (c) root of plantlets; (d) leaf primordial; (e) and (f) flower bud; (g) close-up of leaf; (h) glandular trichome. GST: glandular secretory trichome; TST: T-shaped trichome.

method to study transcripts in trichome cells by quantitative real time PCR (qPCR). Using this method, ADS transcripts were detected in both apical and sub-apical cells of glandular trichomes from A. annua [L. Olofsson et al., unpublished].

The number of GUS-stained GSTs decreased with age of the leaves (Figure 4). A high GST density is observed in young leaves at the apex (Figure 4(a)) while essentially no GSTs are present on old leaves at lowest node (Figure 4(c)). It is quite obvious that the biosynthesis of artemisinin precursors take place in young tissues of $A$. annua.

The activity of the ADS promoter varies temporally and in old tissues essentially no GUS staining could be observed (Figures 3 and 4). It is difficult to quantitatively analyze the expression levels of $A D S$ and GUS controlled by endogenous promoter ( $p A D S-A D S$ ) and the recombinant ADS promoter ( $p A D S-G U S$ ), respectively, in transgenic plants. In order to establish the expression pattern of the two promoters, qPCR was used to analyze the relative expression levels of $p A D S-G U S$ and $p A D S$ $A D S$ in different tissues of transgenic plants setting the expression in stem to 1.0 (Figure 5). The expression pattern of GUS and ADS in aerial parts of the transgenic plant, i.e. flower buds at early or late stages of development and leaf primordia, was essentially the same indicating that the cis-elements controlling glandular trichome-specific expression are included in the cloned promoter. The differences in relative expression levels in these tissues reflect the density of GSTs.

In old leaves and roots at the reproductive stage, the cloned promoter shows considerably higher activity than

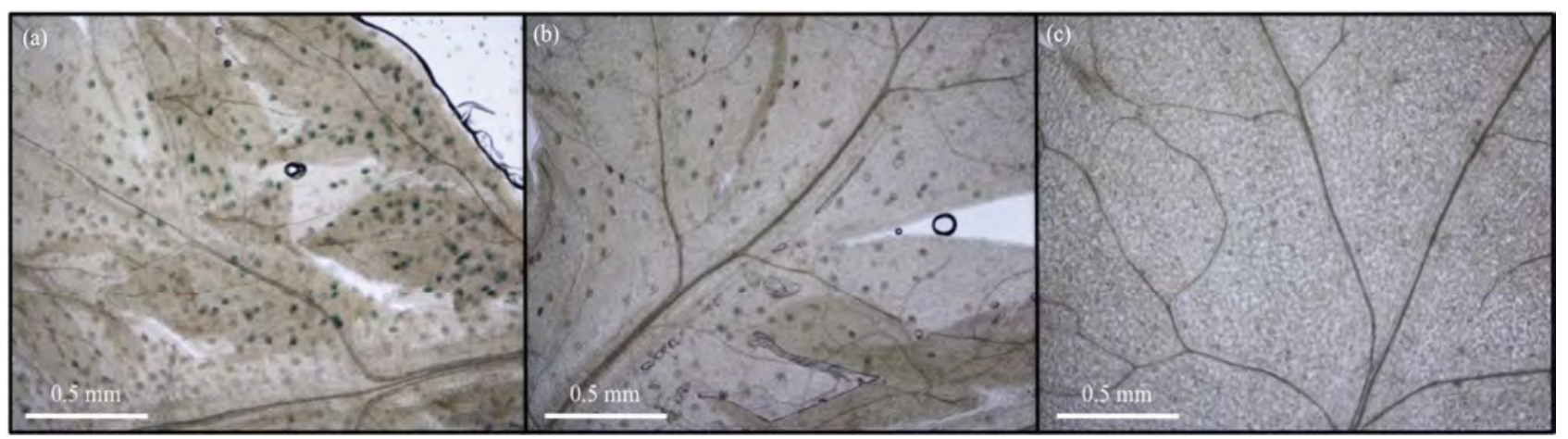

Figure 4. Gus-staining of transgenic A. annua transformed using the pADS-GUS plasmid. a: leaf at apex; b: leaf at node 2; c: old leaf at node 6 . 


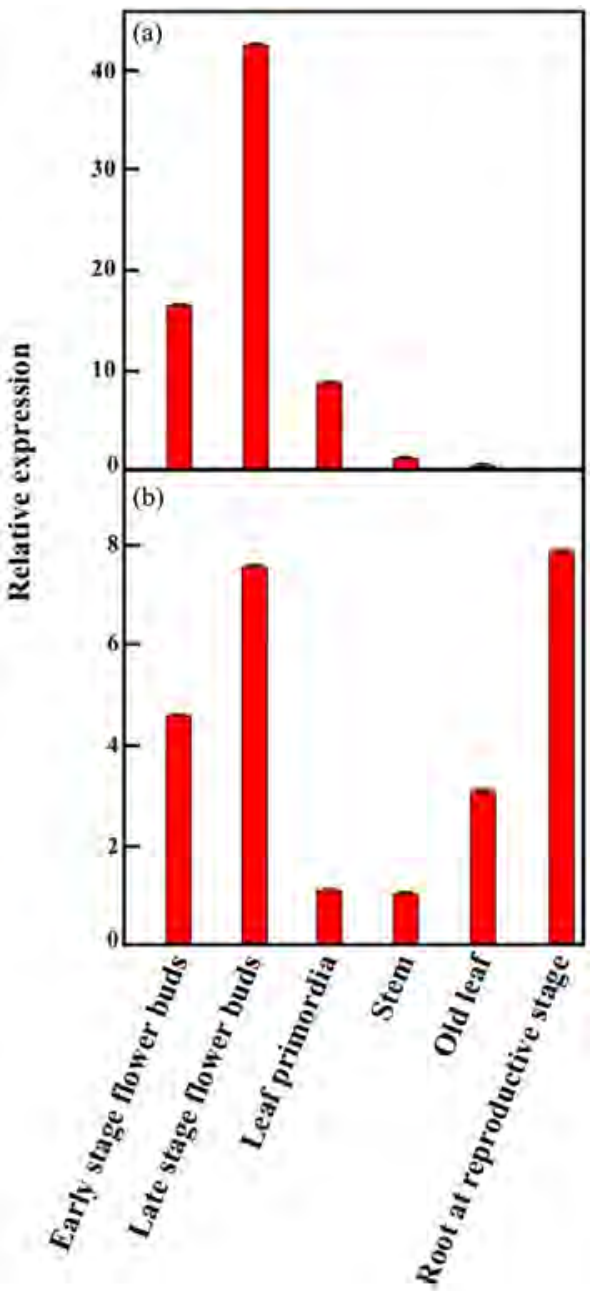

Figure 5. Expression pattern of pADS-ADS (a) and pADSGUS (b) of different tissues. All activities relative to the activity in stem, which was set to 1.0 .

the wild-type promoter (Figure 5). A possible explanation for this observation is that a silencing cis-element controlling ADS expression in these tissues has been deleted in the cloned ADS promoter.

Relative expression levels of $p A D S-G U S$ and $p A D S$ $A D S$ were estimated using the $2^{-\Delta \Delta \mathrm{Ct}}$ method using $\beta$ actin as reference gene [41]. The wild-type promoter showed a considerably higher activity (up to 250-fold) than the recombinant promoter (Figure 6). Apparently, one or more enhancer cis-elements are not included in the cloned ADS promoter. It is possible that such enhancers are located downstream of the ADS start codon, e.g. in an intron as has been reported for other plant genes [42, 43]. In addition to this, the stability of the two mRNAs may differ considerably, i.e. the GUS mRNA is broken down much faster than the ADS mRNA.

The results presented are consistent with ADS being

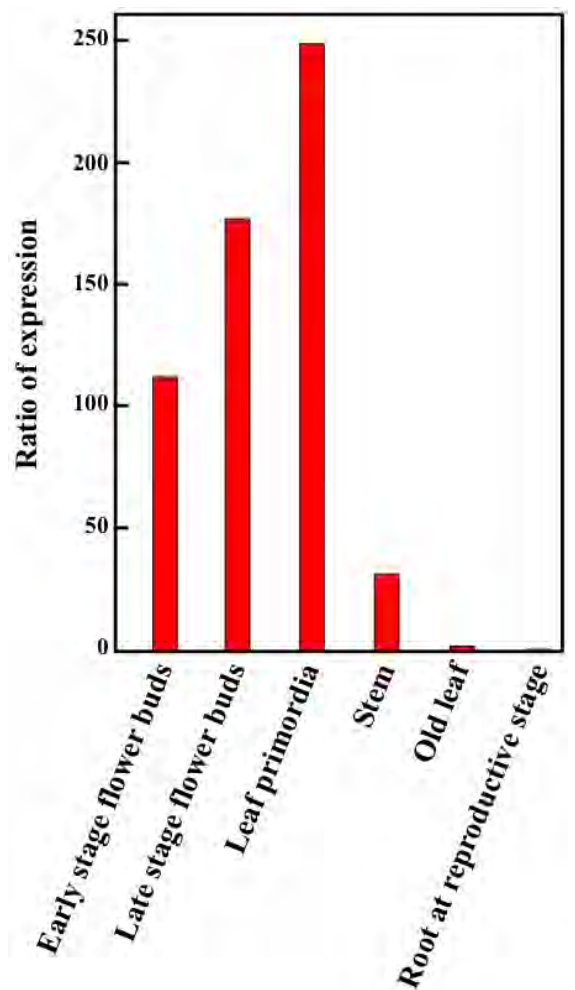

Figure 6. Ratio of expression level of $p A D S-A D S$ to $p A D S$ GUS in aerial tissues of transgenic $A$. annua. For each tissue the expression level for $p A D S-G U S$ was set to 1.0.

specifically located to glandular trichomes and the highest concentration of artemisinin precursors being in upper leaves of A. annua plants [44].

\section{Acknowledgements}

We wish to thank Professor K. Tang, Shanghai Jiao Tong University, for hosting HW during a visit to his laboratory to carry out initial experiments on the genetic transformation of A. annua. We also wish to acknowledge the financial support from the Faculty of Natural Sciences and Engineering, Linnaeus University.

\section{REFERENCES}

[1] D. Rathore, T. F. McCutchan, M. Sullivan and S. Kumar, "Antimalarial Drugs: Current Status and New Developments,” Expert Opinion on Investigational Drugs, Vol. 14, No. 7, 2005, pp. 871-883. doi:10.1517/13543784.14.7.871

[2] T. E. Wallart, N. Pras and W. J. Ouax, "Seasonal Variations of Artemisinin and Its Biosynthetic Precursors in Tetraploid Artemisia annua Plants Compared with the Diploid Wild-Type,” Planta Medica, Vol. 65, No. 8, 1999, pp. 723-728. doi:10.1055/s-1999-14094

[3] N. Delabays, X. Simonnet and M. Gaudin, “The Genetics 
of Artemisinin Content in Artemisia annua L. and the Breeding of High Yielding Cultivars," Current Medicinal Chemistry, Vol. 8, No. 15, 2001, pp. 795-1801.

[4] M. Hommel, "The Future of Artemisinins: Natural, Synthetic or Recombinant?” Journal of Biology, Vol. 7, No. 10, 2008, p. 38. doi:10.1186/jbiol101

[5] F. Jing, L. Zhang, M. Li, Y. Tang, Y. Wang, Y. Wang, Q. Wang, Q. Pan, G. Wang and K. Tang, "Abscisic Acid (ABA) Treatment Increases Artemisinin Content in $\mathrm{Ar}$ temisia annua by Enhancing the Expression of Genes in Artemisinin Biosynthetic Pathway,” Biologia, Vol. 64, No. 2, 2009, pp. 319-323. doi:10.2478/s11756-009-0040-8

[6] T. C. Smith, P. J. Weathers and R. D. Cheetham, "Effects of Gibberellic Acid on Hairy Root Cultures of Artemisia annua: Growth and Artemisinin Production," In Vitro Cellular \& Developmental Biology-Plant, Vol. 33, No. 1, 1997, pp. 75-79. doi:10.1007/s11627-997-0044-4

[7] P. J. Weathers, G. Bunk and M. C. McCoy, “The Effect of Phytohormones on Growth and Artemisinin Production in Artemisia annua Hairy Root," In Vitro Cellular \& Developmental Biology-Plant, Vol. 41, No. 1, 2005, pp. 47-53. doi:10.1079/IVP2004604

[8] Y. S. Zhang, H. C. Ye, B. Y. Liu, H. Wang and G. F. Li, "Exogenous GA3 and Flowering Induce the Conversion of Artemisinic Acid to Artemisinin in Artemisia annua Plants,” Russian Journal of Plant Physiology, Vol. 52, No. 1, 2005, pp. 58-62. doi:10.1007/s11183-005-0009-6

[9] W. Putalun, W. Luealon, W. De-Eknamkul, H. Tanaka and Y. Shoyama, "Improvement of Artemisinin Production by Chitosan in Hairy Root Cultures of Artemisia annua L.,” Biotechnology Letters, Vol. 29. No. 7, 2007, pp. 1143-1146. doi:10.1007/s10529-007-9368-8

[10] A. Baldi and V. K. Dixit, "Yield Enhancement Strategies for Artemisinin Production by Suspension Cultures of Artemisia annua,” Bioresource Technology, Vol. 99, No. 11, 2008, pp. 4609-4614. doi:10.1016/j.biortech.2007.06.061

[11] C. Lei , D. Ma, G. Pu, X. Qiu, Z. Du, H. Wang, G. Li, H. Ye and B. Liu, "Foliar Application of Chitosan Activates Artemisinin Biosynthesis in Artemisia annua L.," Industrial Crops and Products, Vol. 33, No. 1, 2011, pp. 176-182. doi:10.1016/j.indcrop.2010.10.001

[12] L. Zhang, F. Jing, F. Li, M. Li, Y. Wang, G. Wang, X. Sun and K. Tang, "Development of Transgenic Artemisia annua (Chinese Wormwood) Plants with an Enhanced Content of Artemisinin, an Effective Anti-Malarial Drug, by Hairpin-RNA-Mediated Gene Silencing," Biotechnology and Applied Biochemistry, Vol. 52, No. 3, 2009, pp. 199-207. doi:10.1042/BA20080068

[13] L. -L. Feng, R. -Y. Yang, X. -Q. Yang, X. -M. Zeng. W. -J. Lu and Q. -P. Zeng, "Synergistic Re-Channeling of Mevalonate Pathway for Enhanced Artemisinin Production in Transgenic Artemisia annua," Plant Science, Vol. 177, No. 1, 2009, pp. 57-67. doi:10.1016/j.plantsci.2009.03.014

[14] J. -L. Han, B. -Y. Liu, H. -C. Ye, H. Wang, Z. -Q. Li and
G. -F. Li, "Effects of Overexpression of the Endogenouse Farnesyl Diphosphate Synthase on the Artemisinin Content in Artemisia annua L.,” Journal of Integrative Plant Biology, Vol. 48, No. 4, 2006, pp. 482-487. doi:10.1111/j.1744-7909.2006.00208.x

[15] P. Mercke, M. Bengtsson, H. J. Bouwmeester, M. A. Posthumus and P. E. Brodelius, "Molecular Cloning, Expression, and Characterization of Amorpha-4,11-Diene Synthase, a Key Enzyme of Artemisinin Biosynthesis in Artemisia annua L.," Archives of Biochemistry and Biophysics, Vol. 381, No. 2, 2000, pp. 173-180. doi:10.1006/abbi.2000.1962

[16] H. J. Bouwmeester, T. E. Wallaart, M. H. Janssen, B. van Loo, B. J. Jansen, M. A. Posthumus, C. O. Schmidt, J. W. de Kraker, W. A. Konig and M. C. Franssen, "Amorpha-4,11-Diene Synthase Catalyses the First Probable Step in Artemisinin Biosynthesis," Phytochemistry, Vol. 52, No. 5, 1999, pp. 843-854. doi:10.1016/S0031-9422(99)00206-X

[17] S. H. Kim, Y. J. Chang and S. U. Kim, "Tissue Specificity and Developmental Pattern of Amorpha-4,11-Diene Synthase (ADS) Proved by ADS Promoter-Driven GUS Expression in the Heterologous Plant, Arabidopsis thaliana," Planta Medica, Vol. 74, No. 2, 2008, pp. 188-193. doi:10.1055/s-2008-1034276

[18] K. H. Teoh, D. R. Polichuk, D. W. Reed, G. Nowak and P. S. Covello, "Artemisia annua L. (Asteraceae) TrichomeSpecific cDNAs Reveal CYP71AV1, a Cytochrome P450 with a Key Role in the Biosynthesis of the Antimalarial Sesquiterpene Lactone Artemisinin,” FEBS Letters, Vol. 580, No. 5, 2006, pp. 1411-1416. doi:10.1016/j.febslet.2006.01.065

[19] D. -K. Ro, E. M. Paradise, M. Ouellet, K. J. Fisher, K. L. Newman, J. M. Ndungu, K. A. Ho, R. A. Eachus, T. S. Ham, J. Kirby, M. C. Y. Chang, S. T. Withers, Y. Shiba, R. Sarpong and J. D. Keasling, "Production of the Antimalarial Drug Precursor Artemisinic Acid in Engineered Yeast,” Nature, Vol. 440, No. 7, 2006, pp. 940-943. doi:10.1038/nature04640

[20] G. D. Brown and L. K. Sy, "In Vivo Transformations of Dihydroartemisinic Acid in Artemisia annua Plants," Tetrahedron, Vol. 60, No. 5, 2004, pp. 1139-1159. doi:10.1016/j.tet.2003.11.070

[21] Y. Zhang, K. H. Teoh, D. W. Reed, L. Maes, A. Goossens, D. J. Olson, A. R. Ross and P. S. Covello, "The Molecular Cloning of Artemisinic Aldehyde $\Delta 11(13)$ Reductase and Its Role in Glandular Trichome-Dependent Biosynthesis of Artemisinin in Artemisia annua," The Journal of Biological Chemistry, Vol. 283, No. 31, 2008, pp. 21501-21508. doi:10.1074/jbc.M803090200

[22] K. H. Teoh, D. R. Polichuk, D. W. Reed and P. S. Covello, "Molecular Cloning of an Aldehyde Dehydrogenase Implicated in Artemisinin Biosynthesis in Artemisia annua," Botany, Vol. 87, No. 6, 2009, pp. 635-642. doi:10.1139/B09-032

[23] M. V. Duke, R. N. Paul, H. N. Elsohly, G. Sturtz and S. O. Duke, "Localization of Artemisinin and Artemisitene in 
Foliar Tissues of Glanded and Glandless Biotypes of Artemisia annua L.," International Journal of Plant Sciences, Vol. 155, No. 3, 1994, pp. 365-372. doi:10.1086/297173

[24] P. S. Covello, K. H. Teoh, R. Devin, D. R. Polichuk, "Function Genomic and Biosynthesis of Artemisinin," Phytochemistry, Vol. 68, No. 14, 2007, pp. 1864-1871. doi:10.1016/j.phytochem.2007.02.016

[25] L. Olofsson, A. Engström, A. Lundgren and P. E. Brodelius, "Relative Expression of Genes of Terpene Metabolism in Different Tissues of Artemisia annua L.," BMC Plant Biology, Vol. 11, No. 45, 2011, pp. 1-12.

[26] J. Fütterer, A. Gisel, V. Iglesias, A. Klöti, B. Kost, O. Mittelsten Scheid, G. Neuhaus, G. Neuhaus-Url, M. Schrott, R. Shillito, G. Spangenberg and Z. Y. Wang, "Standard Molecular Techniques for the Analysis of Transgenic Plants,” In: I. Potrykus and G. Spangenberg, Eds., Gene Transfer to Plants, Springer Verlag, Berlin, 1995, pp. 215-263.

[27] R. A. Jefferson, T. A. Kavanagh and M. W. Bevan, "GUS Fusions: Beta-Glucuronidase as a Sensitive and Versatile Gene Fusion Marker in Higher Plants," The EMBO Journal, Vol. 6, No. 13, 1987, pp. 3901-3907.

[28] S. Yin, L. Mei, J. Newman, K. Back and J. Chappell, "Regulation of Sesquiterpene Cyclase Gene Expression; Characterization of an Elicitor- and Pathogen-Inducible Promoter," Plant Physiology, Vol. 115, No. 2, 1997, pp. 437-451. doi:10.1104/pp.115.2.437

[29] T. Yang and B. W. Poovaiah, “An Early Ethylene up-Regulated Gene Encoding a Calmodulin-Binding Protein Involved in Plant Senescence and Death,” The Journal of Biological Chemistry, Vol. 275, No. 49, 2000, pp. 38467-38473. doi:10.1074/jbc.M003566200

[30] T. Yang and B. W. Poovaiah, "A Calmodulin-Binding/ CGCG-Box DNA-Binding Protein Family Involved in Multiple Signaling Pathways in Plants," The Journal of Biological Chemistry, Vol. 277, No. 47, 2002, pp. 45049-45058. doi:10.1074/jbc.M207941200

[31] L. D. Zhang, K. J. Zuo, F. Zhang, Y. F. Cao, J. Wang, Y. D. Zhang, X. F. Sun and K. X. Tang, "Conservation of Non-Coding Microsatellites in Plants: Implication for Gene Regulation,” BMC Genomics, Vol. 7, No. 323, 2006, pp. 1-14.

[32] G. -B. Pu, D. -M. Ma, J. -L. Chen, L. -Q. Ma, H. Wang, G. -F. Li, H. -C. Ye and B. -Y. Liu, "Salicylic Acid Activates Artemisinin Biosynthesis in Artemisia annua L.," Plant Cell Reports, Vol. 28, No. 7, 2009, pp. 1127-1135. doi:10.1007/s00299-009-0713-3

[33] D. Ma, G. Pu, C. Lei, L. Ma, H. Wang, Y. Guo, J. Chen, Z. Du, H. Wang, G. Li, H. Ye and B. Liu; "Isolation and Characterization of AaWRKY1, an Artemisia annua Transcription Factor that Regulates the Amorpha-4,11Diene Synthase Gene, a Key Gene of Artemisinin Biosynthesis," Plant and Cell Physiology, Vol. 50, No. 12, 2009, pp. 2146-2161. doi:10.1093/pcp/pcp149
[34] Y. -H. Xu, J. -W. Wang, S. Wang, J. -Y. Wang and X. -Y. Chen, "Characterization of GaWRKY1, a Cotton Transcription Factor that Regulates the Sesquiterpene Synthase Gene $(+)-\delta$-Cadinene Synthase-A,” Plant Physiology, Vol. 135, No. 1, 2004, pp. 507-515. doi:10.1104/pp.104.038612

[35] J. C. Reyes, M. I. Muro-Pastor and F. J. Florencio, "The GATA Family of Transcription Factors in Arabidopsis and Rice,” Plant Physiology, Vol. 134, No. 4, 2004, pp. 1718-1732. doi:10.1104/pp.103.037788

[36] T. Urao, K. Yamaguchi-Shinozaki, S. Urao and K. Shinozaki, "An Arabidopsis myb Homolog Is Induced by Dehydration Stress and Its Gene Product Binds to the Conserved MYB Recognition Sequence,” Plant Cell, Vol. 5, No. 11, 1993, pp. 1529-1539.

[37] R. Solano, C. Nieto, J. Avila, L. Cañas, I. Diaz and J. Paz-Ares, "Dual DNA Binding Specificity of a Petal Epidermis-Specific MYB Transcription Factor (MYB. Ph3) from Petunia hybrida," The EMBO Journal, Vol. 14, No. 8, 1995, pp. 1773-1784.

[38] G. D. Brown, G. -Y. Liang and L. -K. Sy, “Terpenoids from the Seeds of Artemisia annua," Phytochemistry, Vol. 64, No. 1, 2003, pp. 303-323. doi:10.1016/S0031-9422(03)00294-2

[39] J. F. S. Ferreira, J. E. Simon and J. Janick, "Developmental Studies of Artemisia annua: Flowering and Artemisinin Production under Greenhouse and Field Conditions," Planta Medica, Vol. 61, No. 2, 1995, pp. 167-170. doi:10.1055/s-2006-958040

[40] M. E. Olsson, L. M. Olofsson, A. L. Lindahl, A. Lundgren, M. Brodelius and P. E. Brodelius, "Localization of Enzymes of Artemisinin Biosynthesis to the Apical Cells of Glandular Secretory Trichomes of Artemisia annua L.," Phytochemistry, Vol. 70, No. 9, 2009, pp. 1123-1128. doi:10.1016/j.phytochem.2009.07.009

[41] K. J. Livak and T. D. Schmittgen, "Analysis of Relative Gene Expression Data using Real-Time Quantitative PCR and the $2^{-\Delta \Delta C T}$ Method," Methods, Vol. 25, No. 4, 2001, pp. 402-408. doi:10.1006/meth.2001.1262

[42] D. Mascarenhas, I. J. Mettler, D. A. Pierce and H. W. Lowe, "Intron-Mediated Enhancement of Heterologous Gene Expression in Maize,” Plant Molecular Biology, Vol. 15, No. 6, 1990, pp. 913-920. doi:10.1007/BF00039430

[43] M. K. Deyholos and L. E. Sieburth, "Separable WhorlSpecific Expression and Negative Regulation by Enhancer Elements within the AGAMOUS Second Intron," Plant Cell, Vol. 12, No. 10, 2000, pp. 1799-1810.

[44] S. O. Duke and R. N. Paul, "Development an Fine Structure of the Glandular Trichomes of Artemisia annua L.," International Journal of Plant Sciences, Vol. 154, No. 1, 1993, pp. 107-118. doi:10.1086/297096 